\section{International Scientific Journal Theoretical \& Applied Science}

\author{
p-ISSN: 2308-4944 (print) e-ISSN: 2409-0085 (online) \\ Year: 2015 Issue: 05 Volume: 25 \\ Published: $30.05 .2015 \quad$ http://T-Science.org
}

Dossumbek Jakiyayev Associate Professor, Ph.D., Head of the department "Mechanics and Engineering" Taraz State University M.H.Dulati, Kazakhstan ddosumbek1957@mail.ru

Rollan Nussipali

Senior Lecturer, Master

Taraz State University M.H.Dulati, Kazakhstan rollan_n@mail.ru

SECTION 7. Mechanics and machine construction.

\title{
ASSESSMENT OF THE ENERGY CYCLIC STRENGTH MODELS MATERIAL DESTRUCTION
}

\author{
Abstract: The article deals with energy failure criteria under cyclic loading of material, which are \\ summarized in the complex state of stress and transient loading. \\ Key words: destruction of material, energy criteria, cyclic loading. \\ Language: Russian \\ Citation: Jakiyayev D, Nussipali R (2015) ASSESSMENT OF THE ENERGY CYCLIC STRENGTH \\ MODELS MATERIAL DESTRUCTION. ISJ Theoretical \& Applied Science 05 (25): 79-83. \\ Soi: http://s-o-i.org/1.1/TAS*05(25)16 Doi: crossef http://dx.doi.org/10.15863/TAS.2015.05.25.16

\section{УДК 539.385} \section{МАТЕРИАЛА} \\ ОЦЕНКА ЦИКЛИЧЕСКОЙ ПРОЧНОСТИ ПО ЭНЕРГЕТИЧЕСКОЙ МОДЕЛИ РАЗРУШЕНИЯ \\ Аннотация: B статье рассмотрень энергетические критерии разрушения при циклическом \\ нагружении материала, которые обобщены на сложное напряженное состояние и нестационарные \\ режимы нагружения. \\ Ключевые слова: разрушение материала, энергетические критерии, циклическое нагружение.
}

Энергетические представления о развитии деформации и разрушения при циклическом нагружении позволяют более полно описать эти процессы по сравнению с другими подходами (например, деформационными и силовыми). Существующие энергетические критерии циклического разрушения могут быть условно разделены на две основные группы:

1) критерии, основанные на измерении или математическом описании работы пластической деформации, определяемой по петлям гистерезиса;

2) критерии, основанные на учете термодинамических свойств материала и предполагающие аналогию процессов разрушения и плавления.

Первая попытка определить энергию разрушения по петлям гистерезиса была сделана Инглисом, которая показала, что энергия разрушения, вычисленная по петлям, может во много раз превышать энергию статического разрушения, полученную под кривой статического нагружения. Поскольку суммарная энергия, подсчитанная по петлям гистерезиса,функция числа циклов и ассимметрии цикла [1], причем чем больше долговечность, тем больше суммарная энергия, Хансток; а затем Еномото предположили, что на разрушение образца при циклическом деформировании расходуется не вся энергия, а только часть её.

Фентнер и Морроу [2], положившие, что разрушающей энергией в цикле является энергия деформационного упрочнения, впервые попытались выделить разрушающую энергию. Кроме того, они приняли, что суммарная энергия деформационного упрочнения равна энергии, определяемой площадью под кривой статического растяжения в координатах $\sigma_{\text {ист-е. }}$ Ими предложена аналитическая зависимость между амплитудой напряжения $\sigma_{\mathrm{a}}$ и числом циклов до разрушения

$$
\frac{S(1+n)}{2 k}=N_{p}\left(\sigma_{a}\right)^{\frac{1+n}{n}},
$$

где $\mathrm{S}$ - энергия статического растяжения; 
k - коэффициент, определяемый из зависимости $\varepsilon=k\left(\sigma_{a}^{\frac{1}{n}}\right) ; \varepsilon$-деформация;

$\mathrm{n}$ - коэффициент деформационного упрочнения.

Соответствие данной зависимости эксперименту было показано авторами лишь в интервале долговечностей от $10^{5}$ до $10^{6}$ цикл. Уравнение кривой усталости, полученное из (1) имеет вид:

$$
\lg \sigma_{a}=\lg \left[\frac{S(1+n)}{2 k}\right]^{\frac{n}{n+1}}-\frac{n}{1+n} \lg N_{p},
$$

В дальнейшем выражение (1) было упрошено и представлено в виде

$$
\frac{\sigma_{a}}{\sigma_{p}}=\left(\frac{Д_{c y M}}{S}\right)^{-\frac{1}{4}}
$$

где $\sigma_{\mathrm{p}}-$ истинный предел прочности;

Дсум - удельная энергия, рассеянная в материале за цикл.

Уравнение кривой усталости, полученное на основании (3)

$$
\lg \sigma_{a}=\lg \sigma_{\succsim}-\frac{n}{1+5 n} \lg \left(2 N_{p}\right),
$$

Однако проверка критерия (3) показала [3], что лучшее качественное, а в ряде случаев и количественное соответствие дает соотношение, предложенное В.Т. Трощенко и Л.А. Хамаза,

$$
\left(\frac{\sigma_{a}}{\sigma_{\partial}}\right)^{m k+1}=\left(2 N_{p}\right)^{-k},
$$

где $\mathrm{k}$ - постоянная материала, определяемая из эксперимента; $m=\frac{1+n}{n}$ и линейно зависит от логарифма числа циклов нагружения.

Прологарифмировав (5) и сделав приобразавания, получим уравнение кривой усталости

$$
\lg \sigma_{a}=\lg \sigma_{\succsim}-\frac{k}{m k+1} \lg \left(2 N_{p}\right),
$$

Стоуоллом предлагается, что диаграммы деформирования материала как при статическом так и при циклическом нагружении могут быть описаны уравнением [4]:

$$
e=\frac{\sigma_{u c m}}{E}+e_{0} \operatorname{sh} \frac{\sigma_{u c m}}{\sigma_{0}}
$$

где

$\sigma_{0}=\frac{\sigma_{p}-\sigma_{\grave{o}}}{\lg \frac{e_{p}}{0,002}} \quad$ константа;

$e_{0}$ - константа, определяемая экспериментально.

Для пульсирующего цикла нагружения (от 0

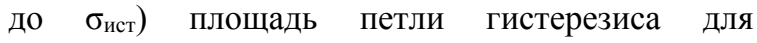

завершающей стадии испытаний на усталость равна

$$
Д=\sigma_{e}-2 \int_{0}^{\sigma_{u c m}} e d \sigma_{u c m},
$$

Поставив (7) в (8), получим

$$
\text { Д }=\sigma_{e} e_{0}\left[\frac{\sigma_{u c m}}{\sigma_{0}} \operatorname{sh} \frac{\sigma_{u c m}}{\sigma_{0}}-\left(2 \operatorname{ch} \frac{\sigma_{u c m}}{\sigma_{0}}-1\right)\right] \text {, }
$$

Учитывая переменность петли гистерезиса в процессе испытания, её среднее значение принимается

$$
Д_{c p}=\frac{1}{2} \text { Д, }
$$

Для случая симметрического цикла

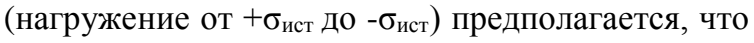
полуцикл сжатия не является опасным. Тогда и в этом случае справедливо уравнение (10). Условие разрушения для пульсирующего цикла нагружения:

$$
N_{p} Д_{c p}=S-\bar{a},
$$

где $\bar{a}$ - энергия, соответствующая напряжению $\frac{1}{2} \sigma_{\text {ист по диаграмме статического }}$ деформирования

$$
\bar{a}=\frac{1}{2} \sigma_{u c m} e_{0} \operatorname{sh} \frac{\sigma_{u c m}}{\sigma_{0}}-\frac{\sigma_{u c m}^{2}}{8 E}-\sigma_{0} e_{0}\left(\operatorname{ch} \frac{\sigma_{u c m}}{2 \sigma_{0}}-1\right),
$$

Для симметричного цикла условие разрушения:

$$
N_{p} Д_{c p}=S,
$$

Окончательные зависимости для числа циклов до разрушения имеют вид:

Для пульсирующего цикла

$$
N_{p}=2 \frac{\frac{\sigma_{p}}{2} \cdot \frac{e_{p}-\sigma_{p} / 2 E}{e_{0}}-\operatorname{ch} \frac{\sigma_{p}}{\sigma_{0}}+1-\frac{\bar{a}}{\sigma_{0} e_{0}}}{\frac{\sigma_{u c m}}{\sigma_{0}} \operatorname{sh} \frac{\sigma_{u c m}}{\sigma_{0}}-2\left(\operatorname{ch} \frac{\sigma_{u c m}}{\sigma_{0}}-1\right)},
$$

Для симметричного цикла

$$
N_{p}=\frac{\frac{\sigma_{p}}{\sigma_{0}} \cdot \frac{e_{p}-\sigma_{p} / 2 E}{e_{0}}-\operatorname{ch} \frac{\sigma_{p}}{\sigma_{0}}+1}{\frac{\sigma_{u c m}}{\sigma_{0}} \operatorname{sh} \frac{\sigma_{u c m}}{\sigma_{0}}-2\left(\operatorname{ch} \frac{\sigma_{u c m}}{\sigma_{0}}-1\right)},
$$

В работе [4] предложено условие усталостого разрушения в виде в виде

$$
N_{p}\left(Д-Д_{r}\right)=Д_{c y м},
$$

где $Д_{r}$ - энергия, необратимо рассеянная в материале за цикл при напряжениях, равных пределу усталости;

$Д_{c y м}-$ предельное значение энергии. 
Зависимость между напряжениями и деформациями принимается:

$$
\varepsilon^{\prime}=\frac{\sigma^{\prime}}{E}+\Delta \varepsilon\left[\frac{2 \sigma^{\prime}}{\Delta \sigma}\right]^{1 / n^{\prime}},
$$

где $\Delta \varepsilon$ - неупругая деформация за цикл;

$\Delta \sigma^{-}$удвоенная амплитуда напряжений за цикл; $\varepsilon^{\prime}, \sigma^{\prime}$ - текущие значение деформации и напряжения.

Площадь петли гистерезиса будет равна

$$
Д=\left(\frac{1-n^{\prime}}{1+n^{\prime}}\right) \Delta \sigma \Delta \varepsilon,
$$

При большом числе циклов до разрушения справедливо уравнение $\quad \Delta \varepsilon N^{k}=\frac{e_{p}}{2^{k}}$, зависимость неупругой деформации за цикл от напряжений может быть представлена в виде $\Delta \varepsilon=R \sigma_{a}^{1 / n^{\prime}}$. Согласно двум последним предположениям получаем зависимость:

$$
\sigma_{a}=\sigma_{p}(2 N)^{-k n^{\prime}},
$$

Сделав преобразования с учетом (18) и (19) получим

$$
N_{p}=\frac{1}{2\left[\left(\frac{\sigma_{a}}{\sigma_{p}}\right)^{\left(1+n^{\prime}\right) / n^{\prime}}-\left(\frac{\sigma_{r}}{\sigma_{p}}\right)^{\left(1+n^{\prime}\right) / n^{\prime}}\right]^{1 / k\left(1+n^{\prime}\right)}}
$$

В работах Д.Е. Мартина и А.В. Муратова $[2,4]$ в качестве критерия усталостного разрушения принимается суммарная энергия упрочнения. Энергия упрочнения за цикл будет

$$
Д_{y n p}=2\left[\frac{1}{2} E_{T}^{u}(\Delta \varepsilon)^{2}\right] \text {, }
$$

За $\mathrm{N}_{\mathrm{p}}$ циклов энергия упрочнения

$$
N_{p} \cdot E_{T}^{u}\left(\Delta \varepsilon^{2}\right)=Д_{c y M},
$$

Мартин принял

$$
Д_{c y M}=\frac{1}{2} E_{T}^{u} e_{p}^{2}
$$

тогда

$$
N_{p} E_{T}^{u}(\Delta \varepsilon)^{2}=\frac{1}{2} E_{T}^{u} e_{p}
$$

или

$$
N^{\frac{1}{2}} \Delta \varepsilon=e_{p} / \sqrt{2}
$$

Уравнение (24) совпадает с уравнением Коффина. Муратовым было найдено, что рассеянная за цикл энергия, связанная с упрочнением, будет равна

$$
Д_{y n p}=\left(\sigma-\sigma_{n u}\right)^{2} \frac{E-E_{T}^{u}}{E \cdot E_{T}^{u}},
$$

Выражение для суммарной рассеянной энергии

$$
Д_{c y s}=Д_{y n p} \cdot N_{p}
$$

На основе этих предположений Муратовым было найдено уравнение кривой усталости и выведена формула для ускоренного определения предела усталости:

$$
\sigma_{r}=\frac{\sigma_{1} \sqrt{N_{1}}-\sigma_{2} \sqrt{N_{2}}}{\sqrt{N_{1}}-\sqrt{N_{2}}},
$$

где $\sigma_{1}, \sigma_{2}$ - напряжения на кривой усталости, соответствующие долговечности $\mathrm{N}_{1}$ и $\mathrm{N}_{2}$.

К числу критериев, основанных на энергии микропластической деформации, относится критерий Гольцева. Д.М. Гольцев [1] рассматривает энергию за цикл Д как результат действия касательных сил неупругого сопротивления, вычисленных с помощью главных и касательных напряжений на октаэдрических площадках:

$$
\text { Д }=в \kappa_{1}\left(\tau_{\text {окт }}+\kappa \sigma_{\text {окт }}\right)^{n} \gamma_{\text {окт }},
$$

где

в- коэффициент, зависящий от формы петли;

$\gamma_{\text {окт }}$ - сдвиговая деформация на октаэдрической площадке;

$\kappa_{1} ;$ к; $\mathrm{n}$ - постоянные.

Разрушение поступает при достижении площадью петли предельного значения

$$
Д=Д_{\text {пред }},
$$

определяемого предельным напряженным состоянием. Для кручения площадь предельной петли может быть выражена (при $\left|\sigma_{1}\right|=\left|\sigma_{3}\right|=\tau_{-1}, \sigma_{2}=0$ ) в виде:

$$
Д_{\tau_{-1}}=\frac{B \kappa_{1}}{G}\left(\frac{\sqrt{6}}{3} \tau_{-1}\right)^{n+1} \text {, }
$$

где G- модуль сдвига.

Приравнивая выражения (28) и (30) выражая величины $\tau_{\text {окт }}$ и $\sigma_{\text {окт }}$ через главные напряжения, получаем условие разрушения при циклическом кручении. Данная работа ценна тем, что в ней сделана попытка распространить энергетические представления о разрушении под действием циклической нагрузки на случай сложного напряженного состояния.

Второй тип критериев основан на предположении, что для нарушения связи атомов твердого тела необходимо вполне определенное количество энергии, независимо от того, как она подводится к телу: механически или в виде тепла.

Исходя из предположения и постоянстве объема при растяжении образца и с учетом 
анализа диаграмм статического растяжения, полученных для малых объемов в зоне образования шейки, А Желимо [1] установил, что предельная работа деформации сталей ферритного и перлитного классов, имеющих близкие значения теплоты плавления, в пределах точности измерения является величиной постоянной. На основании этих исследований была предложена эмпирическая зависимость для вычисления предельной энергии статического разрушения:

$$
A=\frac{\sigma_{e}}{3}\left[\sigma_{T}+2 \sigma_{B}\right]+4,6 \sigma_{B}\left[1+\delta_{e}\right] \lg \frac{1+\delta}{1+\delta_{e}}+\sigma_{B}\left(1+\delta_{e}\right)^{2}\left[\frac{1}{1+\delta}-\frac{1}{1+\delta_{e}}\right]
$$

где $\delta_{e}$ - удлинение на уровне $\sigma_{B} ; \sigma_{T} ; \sigma_{s}^{-}$ соответственно пределы текучести и прочности.

Используя выражения (31) и термодинамические постоянные, Иванова В.С. и Регозин Ю.И. [1] показали на материалах $\mathrm{Cu}, \mathrm{Ta}$, $\mathrm{Al}$, что величины удельный энергии разрушения и энергии плавления имеют один и тот же порядок. На основании этих представлений для малоцикловой усталости была получена следующая зависимость:

$$
\left(\Delta \varepsilon_{p}\right) N^{\frac{1}{2}}=4 \sqrt{\frac{A_{p}}{G}}
$$

являющаяся уравнением Коффина, в котором постоянная определена через удельную энергию разрушения $\mathrm{A}_{\mathrm{p}}$ и модуль сдвига $\mathrm{G}$.

Энергетическая модель разрушения сводится к утверждению, что для разрушения необходима затрата некоторой определенной величины необратимой работы деформирования. Однако сама по себе данная концепция еще недостаточна для инженерных расчетов, так как на накопление повреждений расходуется не вся, а лишь некоторая эффективная доля необратимой работы деформирования [5]. Для установления этой доли, зависящей, по - видимому, от особенностей деформационного процесса, условий теплообмена и т.п., приходиться обращаться к прямым экспериментальным данным. Но все же, в энергетической модели заложены широкие возможности обобщения на сложное напряженное состояние и самые различные режимы нагружения.

В работах $[6,7,8,9]$ предложено уравнение повреждений следующего вида:

$$
\Pi(N)=\frac{\sigma_{\max }(N)}{\bar{\sigma}_{p}}+\sum_{k=1}^{N} \varphi\left(H_{k}, R_{k}\right),
$$

где $k$ - номер соответствующего цикла, N- общее число циклов, $R_{k}$ - текущий коэффициент асимметрии цикла, $\sigma_{\max }(N)^{-}$максимальное за период цикла растягивающее напряжение в момент определения меры повреждения, $\bar{\sigma}_{p}$ - истинное сопротивление разрыву. Безразмерный параметр $H_{k}$, пропорциональный необратимой работе деформирования элемента гипотетического материала, вычисляется по следующей формуле

$$
H_{R}=\omega_{R} \frac{E_{1}}{2 C_{2}^{2}}
$$

Здесь $\omega_{k}{ }^{-}$сумма площадей петель гистерезиса, возникающего в гипотетическом материале за каждый цикл деформирования и $\mathrm{E}_{1}-$ упругая постоянная, которая может быть принята произвольно, так как она не отражается на необратимой работе деформирования. Площадь обратно пропорциональна величине $\mathrm{E}_{1}$, которая должна быть задана при построении графиков деформирования. Таким образом $H_{k}$ от $\mathrm{E}_{1}$ не зависит. Постоянная $\mathrm{C}_{2}$ рассматривается, как абсолютный предел выносливости, отождествляемый в литературе с прецизионным пределом текучести для отдельного кристалического зерна. Эта величина не должна превышать предел ограниченной выносливости материала при симметричном цикле, найденный на такой базе, которая не меньше требуемого pecyрса по числу циклов. Значение $\mathrm{C}_{2}$ рекомендуется принимать равным $(0,6 \ldots 0,8) \sigma_{-1}$.

Связь параметров кинетического уравнения с реальным материалом осуществляется через экспериментальные усталостные кривые, на основании которых строятся экспериментальные графики функции

$$
\varphi(H, R)=\left(1-\frac{\sigma_{\max }}{\bar{\sigma}_{p}}\right) \cdot \frac{1}{N_{p}}
$$

для конкретного материала и определенного типа испытуемых образцов. В этом выражении $\mathrm{N}_{\mathrm{p}}-$ абсцисса усталостной кривой при данном $\mathrm{R}$, отвечающая ординате $\sigma$ мах .

Для вычисления площадей петель пластического гистерезиса необходимо предварительно построить диаграммы деформирования в координатах $\mathrm{S}_{\mathrm{ij}}-\mathrm{e}_{\mathrm{ij}}$, где $\mathrm{S}_{\mathrm{ij}}$ и $\mathrm{e}_{\mathrm{ij}}$ -соответствующие компоненты девиаторов 
напряжений и деформаций. Закон деформирования представлется в виде

$$
E_{i j}=\frac{1+v_{3}}{E_{3}} \cdot S_{i j}+\frac{3}{2} \cdot \frac{\sigma_{i}-C_{2}}{E_{1}} \cdot \frac{S_{i j}}{\sigma_{i}},
$$

где $v_{3}$ и $\mathrm{E}_{3}$ - упругие постоянные, которые однако также, как и $\mathrm{E}_{1}$ на площадях вышеуказанных петель гистерезиса не отражаются. Диаграммы деформирования могут быть принципиально построены при любых, даже нерегулярных режимах нагружения и в таких общих условиях, когда например отдельные компоненты напряжений изменяются несинхронно по произвоьным законам. Проблема сводится к вычислению некоторого приведенного коэффициента асимметрии цикла $\mathrm{R}_{\text {пр }}$, который вносится в уравнение (33) в качестве параметра $\mathrm{R}_{k}$. В том случае, когда такой расчет выполнен, дальнейшее вычисление поврежденности П не вызывает затруднений. При синусоидальных законах изменения отдельных компонентов циклических напражений с одинаковыми периодами рекомендуется следующая формула

$$
R_{n p}=\frac{\Sigma R_{i j} \cdot \omega\left(\sigma_{i j}\right)}{\sum \omega\left(\sigma_{i j}\right)},
$$

где $\omega\left(\sigma_{\mathrm{ij}}\right)-$ площади петель гистерезиса, образующиеся за счет соответствующих компонентов тензора напряжений $\sigma_{\mathrm{ij}}$, $\mathrm{R}_{\mathrm{ij}} \quad-$ коэффициенты асимметрии циклов изменения компонентов $\sigma_{\mathrm{ij}}, \Sigma \omega\left(\sigma_{\mathrm{ij}}\right)$ - сумма площадей всех петель гистерезиса в рассматриваемом цикле нагружения. При некоторых оговорках расчет может проводится также при неодинаковых периодах изменения компонентов напряжений, при бигармонических режимах и в других случаях сложного нагружения.

\section{References:}

1. Romanov AN (1971) Energeticheskie kriterii razrusheniya pri ciklicheskom nagruzhenii. Problemy prochnosti, 1971, №3.

2. Troshhenko VT (1981) Deformirovanie i razrusheniya metallov pri mnogociklovom nagruzhenii. - Kiev: Naukova dumka, 1981.

3. Troshhenko VT, Xamaza LA (1969) Issledovanie nekotoryx energeticheskix kriteriev ustalostnogo razrusheniya metallov. Problemy prochnosti, 1969, №4.

4. Troshhenko VT (1971) Ustalost i neuprugost metallov. - Kiev: Naukova dumka, 1971.

5. Pavlov PA (1980) Mexanicheskie sostoyaniya i prochnost materialov.-L: izdatelstvo LGU, 1980

6. Dzhakiyaev DK, Pavlov PA (1985) Prognozirovanie mnogociklovyx ustalostnyx povrezhdenij stali pri slozhnom neodnorodnom napryazhennom sostoyanii. Tez. dokl. /X vsesoyuznaya nauchno- texn. konf. po konstrukcionnoj prochnosti dvigatelej. Kujbyshev. 1985.
7. Pawlow PA (1983) Ein Energiemodell der hochzyklischen Ermudung und seine praktische Anwendung. Technische Mechanik, 4, 1983, Heft 1, pp.25-33.

8. Pavlov PA, Malibekov AK (1986) Mnogociklovaya ustalost uglerodistyx stalej pri ploskom napryazhennom sostoyanii. Soobshheniya 1 i 2 . Problemy prochnosti, 1986, №6, pp. 55-60, №8, pp 41-45.

9. Malibekov AK, Dzhakiyaev DK (2008) O metodike rascheta dolgovechnosti na osnovy energeticheskoj modeli mnogociklovyx ustalosnyx povrezhdenij. Mexanika i modelirovanie processov texnologii -2008, №2.

10. Serensen SV, Kogaev VP, Shnejderovich RM (1975) Nesushhaya sposobnost i raschety detalej mashin na prochnost. - Moscow: Mashinostroenie, 1975.

11. Burger IA, Shor BF, Iosilevich GB (1979) Raschet na prochnost detalej mashin. Moscow: Mashinostroenie, 1979. 